\title{
Ambiente, estratégia e desempenho em micro e pequenas empresas
}

\author{
Environment, strategy and organizational results in micro and \\ small firms
}

Fábio Mello Fagundes ${ }^{[a]}$, Fernando Antonio Prado Gimenez ${ }^{[b]}$

[a] Bacharel em Sistemas de Informação, Mestre em Administração pela Universidade Positivo (UP), Curitiba, PR - Brasil, e-mail: faggundes@yahoo.com.br

[b] Administrador, Doutor em Administração pela Manchester Business School, professor do Programa de Pós-Graduação em Administração da Pontifícia Universidade Católica do Paraná (PPAD/PUCPR), Curitiba, PR - Brasil, e-mail: fernando.gimenez@pucpr.br

\section{Resumo}

No Brasil, as micro e pequenas empresas representam 99,2\% das 4,1 milhões de empresas formais na indústria, no comércio e nos serviços. A metade dessas empresas fecha antes de completar dois anos de operações. O objetivo deste estudo foi analisar, em micro e pequenas empresas industriais paranaenses, a percepção de turbulência ambiental, a estratégia adotada, o foco estratégico e o desempenho. Além disso, buscou-se verificar a relação entre estratégia e desempenho em diferentes graus de turbulência ambiental. A amostra foi por adesão, com convite por e-mail. Houve 101 respostas, sendo 70 válidas e submetidas a testes estatísticos. Foi utilizado o modelo de Gimenez (1993) para avaliar a turbulência ambiental; o modelo de Miles e Snow (1978) para estratégia; e, para desempenho, o modelo de Kilmann e Herden (1976). Foi detectado o crescimento da tecnologia como fator de pressão ambiental e redução do impacto para as variáveis governo e fornecedores, comparando-se aos resultados das pesquisas de Miles e Snow (1978) e Gimenez (1993). Não foi comprovada estatisticamente a relação entre foco estratégico (lucro ou sobrevivência) e turbulência ambiental. Contudo, foi encontrada relação entre estratégia e objetivo organizacional. O estudo confirmou resultados de trabalhos anteriores e evidenciou a necessidade de se investigar atributos pessoais do estrategista e processos estratégicos em pequenas empresas.

Palavras-chave: Pequena empresa. Estratégia. Turbulência ambiental. 


\begin{abstract}
In Brazil, micro and small firms represent $99.2 \%$ of 4.1 million manufacturing, retail and services firms. Half of these firms stop operating in two years. The aim of this paper was to analyze the perception of environmental turbulence, adopted strategies, strategic focus and organizational results in micro and small firms located in Paraná. The relationship between strategy and organizational results in different degrees of environmental turbulence was, also, analyzed. The convenience sample, invited through electronic mail, comprised 101 firms, from which 70 valid responses were submitted to statistics analyses. The model for evaluation of environmental turbulence proposed by Gimenez (1993) was adopted; for strategy measurement, Miles and Snow's (1978) model was the reference; and Kilmann and Herden's (1976) model of organizational effectiveness was adopted for measuring organizational results. Data analysis indicated the growth of technological changes' relevance as a factor of environmental pressure and reduction of government and suppliers pressures in comparison to what was found by Miles and Snow (1978) and Gimenez (1993). No statistically significant relationship was found between strategic focus (profitability or survival) and environmental turbulence. However, a significant relationship between strategy and organizational objectives was found. The study confirmed previous results and provided evidences of the necessity to research personal attributes of the strategist and strategic processes in small firms.
\end{abstract}

Keywords: Small firm. Strategy. Environmental turbulence.

\section{Introdução}

Os estudos sobre administração estratégica no Brasil têm uma tradição de focar sua atenção em grandes empresas. Na literatura estrangeira, no entanto, o tema "pequenas empresas" há muito é explorado. Robinson e Pearce, em 1984, apresentaram uma classificação das linhas de pesquisa que abordavam a gestão estratégica em pequenas empresas. Gimenez (2000) apontou uma tendência crescente da literatura internacional em tratar do assunto e percebeu o surgimento de uma quinta linha de pesquisa no campo de estudo.

Na última década, o tema parece ter atraído a atenção de pesquisadores brasileiros com maior frequência. Por exemplo, entre 1998 e 2007, é possível encontrar trabalhos que abordaram diferentes aspectos da administração estratégica em pequenas empresas, tais como Gimenez (1998), Gimenez et al. (1999), Barros (2001), Cochia e Machado-da-Silva (2004), Cavedon e Ferraz (2005), Hayashi Jr., Baraniuk e Bulgacov (2006), Rossetto et al. (2006) e Santos, Alves e Almeida (2007). Esses estudos abordaram questões relacionadas a conteúdo e processo estratégico em micro e pequenas empresas em diferentes contextos ambientais. No seu conjunto, os estudos anteriores apontam para a necessidade de verificar, de forma mais abrangente, como se dá a relação entre escolhas estratégicas, contexto ambiental e desempenho em pequenas empresas.
A preocupação com estudos em pequenas empresas, no nível estratégico, se justifica principalmente pelo fato de que há especificidades da gestão nas pequenas empresas, diferenciando-as das grandes, o que leva à necessidade de modelos conceituais apropriados a esse tipo de empresa, como foi bem argumentado por Cooper (1981). Além disso, os estudos sobre gestão de pequenas empresas, em geral, se justificam pela contribuição que as micro e pequenas empresas (MPEs) fazem em termos econômicos. As MPEs representam 20\% do Produto Interno Bruto (PIB) nacional, representam, também, $99,2 \%$ das 4,1 milhões de empresas formais na indústria, no comércio e nos serviços e empregam $45 \%$ da força de trabalho que possui carteira assinada. Contudo, a metade dessas empresas fecha antes de completar dois anos de operações, $57 \%$ não passam do terceiro ano e $60 \%$, do quarto ano de existência (SERVIÇOBRASILEIRO DE APOIO ÀS MICRO E PEQUENAS EMPRESAS - SEBRAE, 2007). Esses dados indicam a necessidade de se estudar a gestão estratégica no âmbito de empresas de pequeno porte, buscando explicações mais abrangentes e que permitam contribuir para a diminuição dessas taxas de fracasso (MACHADO, 2007).

O estudo relatado neste artigo teve por objetivo analisar as principais pressões ambientais notadas pelos gestores de MPEs industriais paranaenses, identificar estratégias, objetivos, desempenho e a relação entre estratégia e desempenho 
organizacional em diferentes graus de turbulência ambiental. A intenção do estudo foi contribuir na discussão sobre a interferência do ambiente na relação entre estratégia e desempenho. $\mathrm{O}$ trabalho encontra-se estruturado em mais quatro seções, além desta introdução. A seguir, apresenta-se o referencial teórico que apoiou este estudo. Após a discussão do referencial teórico, os procedimentos de pesquisa são explicados. Depois disso, os resultados do estudo são demonstrados e analisados. Por fim, conclui-se o trabalho com a indicação de sua contribuição para este campo de estudo.

\section{Estratégia, contexto ambiental e desempenho em pequenas empresas}

As principais correntes teóricas sobre estratégias competitivas enfatizam o desenvolvimento e a verificação de teorias em um contexto no qual as firmas, geralmente de grande porte, agem no sentido de maximizar o lucro (BANDEIRA-DE-MELLO; CUNHA, 2004). Ansoff (1990, p. 47) também ressalta a importância do lucro ao afirmar que "para uma empresa, a questão do lucro é de grande importância como indicador econômico de eficiência".

Contudo, Bandeira-de-Mello e Cunha (2004), ao proporem uma teoria da administração de risco, afirmam que, em ambientes turbulentos e com fortes pressões governamentais, as pequenas empresas tendem a abdicar do lucro para focar sua estratégia na sobrevivência. O objetivo de Bandeira-de-Mello e Cunha (2004) foi desenvolver uma teoria substantiva da formação de estratégia em pequenas empresas, com base no método da grounded theory, para explicar o comportamento estratégico de pequenas empresas em ambientes de forte turbulência e influência governamental. Em sua análise, os autores afirmam que:

$\mathrm{O}$ relacionamento entre pequenas empresas e atores ambientais em um ambiente turbulento e com forte influência governamental, pode ser explicado por um processo social, no qual firmas, com pouca capacidade de influenciar o ambiente em proveito próprio, lidam com adversidades impostas a elas pelos atores ambientais e agem no sentido de maximizar sua longevidade em detrimento da maximização dos ganhos econômico-financeiros (BANDEIRA-DE-MELLO; CUNHA 2004, p. 159).
A turbulência ou incerteza ambiental já foi abordada em muitos estudos (MILLIKEN, 1987; JAUCH; KRAFT, 1986; DOWNEY et al., 1975; MILES; SNOW, 1978). Em geral, seja se referindo à turbulência ambiental ou à incerteza ambiental, os autores a conceituam como a dificuldade de antever as mudanças ambientais a que as empresas estão expostas. Turbulência (ou incerteza) tem sido um conceito central na literatura de teoria organizacional, particularmente nas teorias que procuram explicar a relação entre organizações e seus ambientes (MILLIKEN, 1987; THOMPSON, 1967).

Os três aspectos mais comuns para definir incerteza ambiental foram organizados por Milliken (1987), a partir de diversos autores:

a) inabilidade em notar probabilidades de futuros eventos bons;

b) falta de informação sobre a relação causa e efeito;

c) inabilidade de predizer os resultados de uma decisão.

Milliken (1987) propôs a existência de três tipos de incertezas ambientais: estado de incerteza, efeito incerteza e resposta incerta. O estado de incerteza é quando a previsão do ambiente ou parte dele se torna muito difícil. Já o efeito de incerteza está relacionado com a dificuldade de predizer o futuro em virtude do efeito de uma mudança no ambiente de forma inesperada, como, por exemplo, um furacão ou um tufão. $\mathrm{O}$ terceiro tipo de incerteza, a resposta incerta, está relacionado à inabilidade de predizer as consequências das escolhas feitas por parte dos gestores.

Jauch e Kraft (1986) consideram que a incerteza ambiental tem algumas dimensões: disparidade (heterogeneidade), volatilidade, quantidade das mudanças e velocidade em que ocorrem e predisposição a elas. Downey et al. (1975) comentam que alguns estudos sugerem que diferenças individuais são o motivo da variação da percepção de incerteza. Para Gimenez (1993), a forma como uma empresa tenta se adaptar às mudanças no ambiente está relacionada à visualização destas como ameaças ou oportunidades. Ramos et al. (2005) lembram que o aumento da velocidade das mudanças, em razão da globalização, tem causado um aumento da turbulência ambiental. Já Corrêa e Prochno (1998) destacam que no cenário brasileiro as empresas devem atentar-se às 
mudanças e responder de forma rápida ao ambiente. Bandeira-de-Mello e Cunha (2004) enfatizam que, no caso do Brasil, a incerteza ou (turbulência) ambiental não se dá somente por conta da dinâmica competitiva (inovações tecnológicas e novos entrantes), mas pelos efeitos da atuação de governos não facilitadores, que são fracos na implementação de leis e principalmente hostis aos empreendedores.

Para Ansoff (1990), estratégia é um dos vários conjuntos de regras de tomada de decisão para orientar o comportamento de uma organização. Mintzberg e Quinn (2001) discutem o conceito de estratégia e apresentam cinco definições, que recebem o nome de $5 \mathrm{Ps}^{1}$ da estratégia: a estratégia como plano é uma direção, um guia ou curso de ação para o futuro, um caminho para ir daqui a ali, ou seja, é a definição dos líderes em relação ao que se pretende a longo prazo; a estratégia como padrão é a consistência em comportamento ao longo do tempo, analisando o comportamento passado para definir seus "padrões"; a estratégia como posição é a localização de determinados produtos em determinados mercados; a estratégia como perspectiva é a maneira fundamental de uma organização fazer as coisas, olhando para dentro da organização em algo chamado de "teoria do negócio"; a estratégia como pretexto é uma "manobra” específica para enganar um oponente ou concorrente.

Porter (2002) diz que uma das melhores formas de saber se uma empresa tem estratégia é verificar se tomou decisões claras a respeito do que não pretende fazer. Ainda que o executivo goste muito de enumerar em listas intermináveis as coisas que faz, do ponto de vista estratégico é mais importante ter uma lista das coisas que não quer fazer. A estratégia não consiste em satisfazer todas as necessidades dos clientes. É preciso entender que estes são diferentes e que as empresas não podem atender a todos com a mesma dedicação e entusiasmo, pois, se o fizer, perderá o foco, tendendo a ver todos com o mesmo valor. Agir estrategicamente é deixar alguns clientes insatisfeitos para que outros possam ficar verdadeiramente contentes.

De acordo com Miles e Snow (1978), as empresas desenvolvem padrões de comportamento estratégico relativamente estáveis na busca de um bom alinhamento com as condições ambientais observadas pela administração. Esses padrões foram categorizados em quatro estratégias genéricas apresentadas no Quadro 1. A base do trabalho de Miles e Snow centra-se em três idéias, conforme Gimenez (2000, p. 32):

a) o ambiente dá forma e é formado pelas ações organizacionais - construção do ambiente;

b) escolhas estratégicas feitas pela administração da empresa dão forma à estrutura e aos processos organizacionais;

c) processos e estrutura condicionam a estratégia.

\section{Categoria \\ estratégica \\ Estratégia Uma empresa seguindo essa estratégia procura localizar e manter uma linha de produtos/serviços relativamente defensiva estável. Seu foco concentra-se em uma gama de produtos/serviços mais limitada do que seus concorrentes e tenta proteger seu domínio por meio da oferta de produtos com melhor qualidade, serviços superiores e/ ou menores preços. Não procura estar entre os líderes da indústria, restringindo-se àquilo que sabe fazer tão bem ou melhor que qualquer um. \\ Estratégia Uma empresa que adota essa estratégia está continuamente ampliando sua linha de produtos/serviços. Enfatiza prospectora a importância de oferecer novos produtos/serviços em uma área de mercado relativamente mais ampla. Valoriza ser uma das primeiras a oferecer novos produtos, mesmo que todos os esforços não se mostrem altamente lucrativos.}

Quadro 1 - Categorias estratégicas de Miles e Snow

(Continua)

1 Henry Mintzberg publicou pela primeira vez o conceito dos 5 Ps da estratégia no California Management Review, em 1987. 


\begin{abstract}
Categoria
estratégica

Descrição

Estratégia

Uma empresa que segue essa estratégia tenta manter uma linha limitada de produtos/serviços relativamente analítica estável e ao mesmo tempo tenta adicionar um ou mais novos produtos/serviços que foram bem-sucedidos em outras empresas do setor. Em muitos aspectos é uma posição intermediária entre as estratégias defensiva e prospectora.

Estratégia reativa

A firma que adota uma estratégia reativa exibe um comportamento mais inconsistente do que os outros tipos. É uma espécie de não estratégia. Não arrisca em novos produtos/serviços a não ser quando ameaçada por competidores. A abordagem típica é "esperar para ver" e responder somente quando forçada por pressões competitivas para evitar a perda de clientes importantes e/ou manter a lucratividade.
\end{abstract}

Quadro 1 - Categorias estratégicas de Miles e Snow

(Conclusão)

Fonte: Adaptado de DAVIG, 1986, p. 42.

Essa taxonomia tem sido utilizada em diversos estudos com pequenas empresas no Brasil. Rossetto et al. (2006) encontraram uma tendência de adoção da estratégia analítica por pequenas pousadas na cidade de Florianópolis, com um processo adaptativo de formulação da estratégia. Cochia e Machado-da-Silva (2004) revelaram que diferentes posicionamentos competitivos, com base nas categorias de Miles e Snow (1978) foram adotados por dirigentes de pequenas empresas industriais dos setores de vestuário e alimentos. Além disso, os resultados deram suporte para as perspectivas institucional e cognitiva da estratégia, ao evidenciarem que esses diferentes posicionamentos se davam em contextos ambientais muito semelhantes. Cavedon e Ferraz (2005) estudaram pequenos comércios na cidade de Porto Alegre e descobriram que as representações sociais do ambiente enfrentado pelos estrategistas têm relação com diferentes posturas estratégicas. Por exemplo: uma visão de futuro baseada no comodismo associou-se a uma postura reativa, enquanto que o desejo de mudança, com cautela, associou-se à postura defensiva.

A definição de desempenho é tão complexa quanto a de estratégia. Ao mesmo tempo, é tão fundamental quanto esta, pois não faz sentido estudar estratégia sem ter como objetivo atingir um bom desempenho (PLESHKO, 2007). A primeira noção que nos vem à cabeça ao pensar em desempenho de organizações éa de que esse conceito é uma variável econômica, pois uma empresa, em um mundo capitalista, tem como objetivo principal dar lucro (PAULA, 2006). Contudo, Kilmann e Herden (1976) propuseram um modelo de avaliação de desempenho organizacional por meio da satisfação com o objetivo organizacional da empresa.
Esse modelo levou em consideração o modelo de Jung de tipos psicológicos, que consiste na relação entre a percepção e o julgamento. Conforme essa teoria, a percepção é dividida em sensibilidade ou intuição e o julgamento é dividido em pensar ou sentir. Kilmann e Herden (1976) definiram quatro tipos de objetivos organizacionais, conforme se verifica no Quadro 2, e operacionalizados, de acordo com o Quadro 3.

Gimenez (1993), adotando a classificação de objetivos organizacionais do modelo de Kilmann e Herden (1976), questionou os empresários quanto ao grau de satisfação pessoal com o nível de atingimento de seus objetivos. Isso se deu por meio de uma escala do tipo Likert $(1=$ muito baixo; $2=$ baixo; $3=$ mediano; 4 = alto; 5 = muito alto). Essa medida foi tomada como substituta às medidas de desempenho da empresa, tendo em vista a notória falta de exatidão nos registros de desempenho de pequenas empresas. Nesta pesquisa, o objetivo mais importante para os respondentes $(50 \%)$ foi a eficiência interna, e também mereceu destaque a eficácia externa $(27 \%)$.

\section{Procedimentos de pesquisa}

Como o objetivo foi estudar a estratégia em micro e pequenas empresas, foi adotada a classificação de micro e pequenas empresas industriais proposta pelo SEBRAE, ou seja, o estudo restringiuse às empresas com até 99 empregados. Para uma análise mais abrangente da indústria como um todo, optou-se por um enfoque multissetorial. A região de aplicação da pesquisa foi o Estado do Paraná, Brasil. 
A abordagem metodológica escolhida foi quantitativo-descritiva de corte transversal, ou seja, uma investigação empírica cuja finalidade principal foi o delineamento ou a análise de fatos ou fenômenos.

Os gestores das MPEs industriais paranaenses foram convidados a participar da pesquisa, que ficou disponível na internet. A amostragem se deu por forma de adesão à pesquisa, por meio de contato por e-mail. Os respondentes foram selecionados a partir do cadastro da Federação das Indústrias do Estado do Paraná (FIEP) 2006 e 2007. Em uma primeira etapa, a partir das 5.048 empresas com menos de 100 funcionários cadastradas em 2006, selecionaram-se as que tinham e-mail no cadastro. Com isso, foram enviadas 4.087 mensagens de correio eletrônico. Em uma segunda etapa, foram selecionadas as empresas presentes no cadastro da FIEP de 2007 com e-mail cadastrado e que não estavam na listagem de 2006. Dessa forma, foram enviados mais 1.110 e-mails. Quinze por cento das mensagens enviadas foram devolvidas porinexistência de endereço. Uma segunda rodada de mensagens foi efetuada 15 dias após.

\section{Objetivo}

Descrição

Eficiência interna Maximizar a eficiência do negócio com o maior volume de vendas possível ao menor custo.

Eficiência externa Maximizar a habilidade da firma em adquirir recursos de outras organizações e distribuir produtos e serviços aos clientes.

Eficácia interna Maximizar a motivação e o envolvimento dos empregados para com suas tarefas.

Eficácia externa Maximizar o nível de satisfação da sociedade para com a empresa (sociedade inclui comunidade, fornecedores e consumidores).

Quadro 2 - Objetivos organizacionais

Fonte: HOY; HELLRIEGEL, 1982, p. 315.

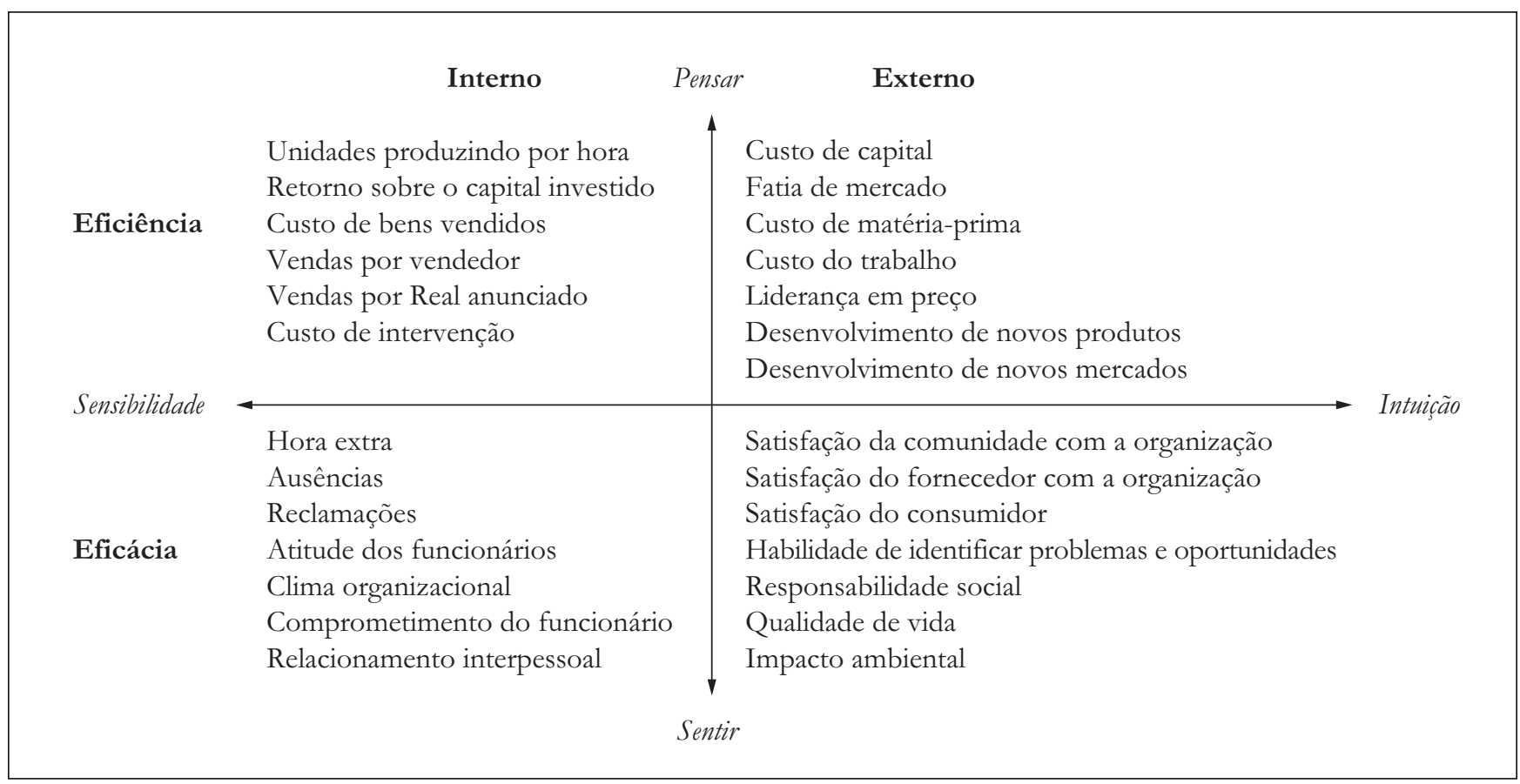

Quadro 3 - Operacionalização do modelo de Kilmann e Herden

Fonte: Adaptado de KILMANN e HERDEN, 1976, p. 95.

REBRAE. Revista Brasileira de Estratégia, Curitiba, v. 2, n. 2, p. 133-146, maio/ago. 2009 
Ao final, foram obtidas 101 respostas, das quais 9 avisaram que não eram empresas industriais e foram desconsideradas. Além disso, 20 respostas vieram de médias empresas e duas foram descartadas por terem sido respondidas por pessoas do nível operacional da empresa. Dessa forma, a amostra final foi de 70 empresas.

A coleta dos dados foi feita por meio de um sítio da internet, o qual os respondentes acessavam por meio de instruções enviadas junto com o convite para participar da pesquisa. O questionário continha 10 questões, sendo utilizado o modelo de Gimenez (1993) para avaliar a turbulência ambiental; o modelo de Miles e Snow (1978), na aplicação de Davig (1986), para estratégia; para foco estratégico (lucro ou sobrevivência), foram criadas perguntas específicas; e, para desempenho, o modelo de Kilmann e Herden (1976).

A análise dos dados foi feita de forma interpretativa, partindo da análise das relações existentes. Para tanto, foi usado, como ferramenta base, o software Microsoft ${ }^{\circledR}$ Excel 2002, onde os dados foram tratados, categorizados e as variáveis foram relacionadas. Para fortalecer as conclusões do estudo, as análises que envolviam relações entre variáveis foram submetidas ao teste Qui-quadrado para verificar a significância dos resultados.

\section{Resultados}

Na Tabela 1, são apresentadas as características gerais da amostra, composta por micro e pequenas empresas, com $88,6 \%$ delas com mais de cinco anos de existência, indicando predominância na amostra de empresas consolidadas, e a existência apenas de respondentes em nível tático-gerencial.

Quanto ao ambiente, os respondentes foram solicitados a classificar sua percepção das transformações de sete variáveis ambientais, utilizando uma escala de 1 a 5 , conforme descrito na Tabela 2. Esse procedimento permitiu identificar quais as variáveis mais impactantes para as empresas estudadas, conforme Gráfico 1.

As pressões ambientais que mais impactaram as MPEs industriais paranaenses foram a tecnologia, a intensidade da concorrência e os hábitos do consumidor. Esse resultado mostra o grande crescimento da tecnologia como pressão ambiental comparando-se a estudos anteriores, como Miles e Snow (1978), que nem ao menos citavam tecnologia, e Gimenez (1993), que apresentava tecnologia como o sexto item de maior impacto para as organizações. Outro item que demonstrou ser fonte de preocupação foi a concorrência, que, nos estudos de Miles e Snow (1978), apareceu em primeiro lugar e, no estudo de Gimenez (1993), em quinto. Já as variáveis ambientais "governo" e "fornecedores" demonstraram queda se comparadas com o estudo de Gimenez (1993).

A estratégia mais aplicada nas MPEs industriais paranaenses foi a prospectora, que esteve presente em 48,6\% das empresas. Em seguida, surgiu a defensiva, com $30,0 \%$, a analítica, com $18,6 \%$, e a menos adotada foi a reativa, com $2,9 \%$. Em comparação com o estudo de Gimenez (1993), ficou claro um crescimento na utilização de estratégias prospectoras e defensivas e uma redução das estratégias analíticas e reativas. A redução na utilização da estratégia reativa pode ser um sinal da estabilização econômica brasileira dos últimos anos, o que faz com que as empresas tenham maior tranquilidade em relação ao seu ambiente e assim sintam-se mais propensas a planejar. A Tabela 3 apresenta os resultados do atual estudo, contrastando-os com os do realizado por Gimenez em 1993.

Tabela 1 - Características da amostra

\begin{tabular}{lccc}
\hline Variável & Categoria & $\begin{array}{c}\text { Quantidade de } \\
\text { respondentes }\end{array}$ & \% \\
\hline $\begin{array}{l}\text { Quantidade de } \\
\text { funcionários }\end{array}$ & $1-19$ & 26 & 37,1 \\
\hline Idade da & $20-99$ & 44 & 62,9 \\
empresa & $01-05$ & 8 & 11,4 \\
& $06-10$ & 19 & 27,1 \\
& $11-20$ & 29 & 41,4 \\
& $>20$ & 14 & 20,0 \\
\hline Nível dos & Direção & 50 & 71,4 \\
respondentes & Gestão & 12 & 17,1 \\
& Coordenação & 8 & 11,4 \\
\hline & Total geral & 70 & 100 \\
\hline
\end{tabular}

Fonte: Dados da pesquisa. 
Tabela 2 - Percepção das pressões ambientais por média

\begin{tabular}{lccc}
\hline $\begin{array}{l}\text { Pressões } \\
\text { ambientais }\end{array}$ & Média & Moda & Desvio-padrão \\
\hline Tecnologia & 4,26 & 5 & 0,90 \\
Concorrência & 4,06 & 4 & 0,99 \\
Consumidor & 3,89 & 4 & 0,99 \\
Mercado & 3,56 & 4 & 1,20 \\
financeiro & 3,34 & 4 & 1,06 \\
Fornecedores & 3,33 & $3 / 4$ & 1,18 \\
Governo & 2,97 & 2 & 1,31 \\
Mão de obra & &
\end{tabular}

Fonte: Dados da pesquisa.

Legenda: Respostas possíveis: 1 = nenhuma mudança; 2 = poucas mudanças pouco importantes; $3=$ muitas mudanças pouco importantes; $4=$ algumas mudanças importantes; $5=$ muitas mudanças importantes.

Um dos objetivos deste estudo foi compreender o que se denominou "foco estratégico", ou seja, o que a empresa estava objetivando com sua estratégia. Os focos estratégicos categorizados nesta pesquisa foram: lucro ou sobrevivência.

Para esta análise, decidiu-se questionar os respondentes de duas maneiras. Em primeiro lugar, de forma "indireta", o questionário continha uma questão que direcionava, de forma oculta ao respondente, a essas duas possibilidades. Além disso, uma segunda pergunta, efetuada de forma direta, colocava as duas alternativas para o respondente.

Ao serem questionados de forma indireta, 90\% dos respondentes demonstraram focar sua estratégia no lucro e 10\% na sobrevivência. Já na forma direta, $64,3 \%$ dos respondentes demonstraram focar a estratégia na sobrevivência e 35,7\%, no lucro. Para melhor análise, as informações das duas questões foram agrupadas, conforme se observa na Tabela 4. Os respondentes que preencheram "lucro" nas duas questões foram classificados com foco nolucro, os que preencheram "sobrevivência" nas duas foram classificados como sobrevivência e, por fim, os que responderam "lucro" em uma e "sobrevivência" em outra ficaram com o foco lucro/ sobrevivência.

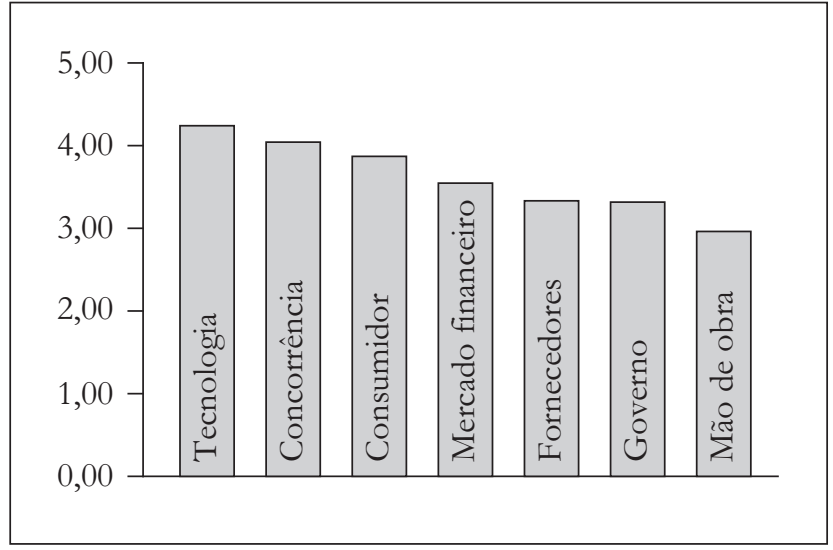

Gráfico 1 - Pressões ambientais Fonte: Dados da pesquisa.

Tabela 3 - Estratégia

\begin{tabular}{lrrrr}
\hline Estratégia & \multicolumn{2}{c}{$\begin{array}{l}\text { Quantidade de } \\
\text { respondentes }\end{array}$} & \multicolumn{2}{c}{$\begin{array}{c}\text { Distribuição no } \\
\text { estudo de Gimenez } \\
\text { (1993) }\end{array}$} \\
\hline Prospectora & 34 & $(48,6 \%)$ & 31 & $(43,7 \%)$ \\
Defensiva & 21 & $(30,0 \%)$ & 15 & $(21,1 \%)$ \\
Analítica & 13 & $(18,6 \%)$ & 20 & $(28,2 \%)$ \\
Reativa & 2 & $(2,9 \%)$ & 5 & $(7,0 \%)$ \\
Total geral & 70 & & 71 & \\
\hline
\end{tabular}

Fonte: Dados da pesquisa.

Tabela 4 - Foco estratégico

\begin{tabular}{lcc}
\hline Foco estratégico & $\begin{array}{c}\text { Quantidade de } \\
\text { respondentes }\end{array}$ & $\mathbf{\%}$ \\
\hline Lucro & 23 & 32,9 \\
Lucro/sobrevivência & 42 & 60,0 \\
Sobrevivência & 5 & 7,1 \\
\hline
\end{tabular}

Fonte: Dados da pesquisa.

O foco estratégico foi relacionado com a estratégia. Os resultados demonstram que as estratégias mais escolhidas pelos respondentes, que foram prospectora e defensiva, apresentam diferentes focos estratégicos. A estratégia defensiva obteve $42,9 \%$ de 
foco nolucro, enquanto a prospectora obteve $32,4 \%$. Contudo, estatisticamente, não se pode afirmar que existe relação entre estratégia e foco estratégico, pois o teste Qui-quadrado não demonstrou diferenças significativas (Tabela 5).

O desempenho foi medido utilizando o modelo de Kilmann e Herden (1976), por meio do qual os respondentes foram solicitados a escolher qual objetivo organizacional mais lhes interessava e a satisfação com o atingimento deste. O objetivo mais importante para 55,7\% dos respondentes foi a eficiência interna, enquanto para $31,4 \%$ foi a eficácia externa. A eficácia interna obteve $11,41 \%$ e a eficiência externa obteve 1,4\%. Essa distribuição foi comparada com o estudo anterior de Gimenez (1993). Houve uma diminuição de respondentes com eficiência externa. Contudo, por meio do teste Qui-quadrado, não houve diferenças significativas entre os dois estudos (Tabela 6).

A satisfação com o objetivo organizacional foi medida por meio de uma escala do tipo Linkert $(1=$ muito baixo; $2=$ baixo; $3=$ mediano; $4=$ alto; $5=$ muito alto). Esses valores foram divididos em três categorias: de 1 a $2=$ insatisfeito; $3=$ regular; e 4 a $5=$ satisfeito. A maioria dos respondentes apresentou um grau regular de satisfação com seu objetivo: 50\%. Já 40\% disseram estar satisfeitos com seu objetivo e $10 \%$ insatisfeitos, conforme demonstrado na Tabela 7.

Tabela 5 - Correlação estratégica versus foco estratégico

\begin{tabular}{|c|c|c|c|c|c|c|c|c|}
\hline \multirow[b]{3}{*}{ Lucro } & \multicolumn{8}{|c|}{ Estratégia } \\
\hline & \multicolumn{2}{|c|}{ Prospectora } & \multicolumn{2}{|c|}{ Defensiva } & \multicolumn{2}{|c|}{ Analítica } & \multicolumn{2}{|c|}{ Reativa } \\
\hline & $11 *$ & $(32,4 \%)^{*}$ & $9 *$ & $(42,9 \%)^{*}$ & $2 *$ & $(15,4 \%)^{*}$ & 1 & $(50,0 \%)$ \\
\hline Lucro/sobrevivência & $22 *$ & $(64,7 \%)^{*}$ & $10^{*}$ & $(47,6 \%)^{*}$ & $10^{*}$ & $(76,9 \%)^{*}$ & & \\
\hline Sobrevivência & 1 & $(2,9 \%)$ & 2 & $(9,5 \%)$ & 1 & $(7,7 \%)$ & 1 & $(50,0 \%)$ \\
\hline Totais & 34 & & 21 & & 13 & & 2 & \\
\hline \multicolumn{9}{|c|}{ Teste Qui-quadrado } \\
\hline Graus de liberdade (df) & & 2 & & ralor & & 212 & & \\
\hline
\end{tabular}

Fonte: Dados da pesquisa.

Legenda: $*$ o teste foi realizado com este grupo.

Tabela 6 - Objetivo organizacional

\begin{tabular}{|c|c|c|c|c|}
\hline \multirow{2}{*}{$\begin{array}{l}\text { Objetivo organizacional } \\
\text { Eficiência interna }\end{array}$} & \multicolumn{2}{|c|}{ Quantidade de respondentes } & \multicolumn{2}{|c|}{ Distribuição estudo Gimenez (1993) } \\
\hline & $39 *$ & $(55,7 \%)^{*}$ & $24 *$ & $(47,1 \%)^{*}$ \\
\hline Eficácia externa & $22 *$ & $(31,4 \%) *$ & $14^{*}$ & $(27,5 \%) *$ \\
\hline Eficácia interna & $8^{*}$ & $(11,4 \%)^{*}$ & $6^{*}$ & $(11,8 \%)^{*}$ \\
\hline Eficiência externa & $1 *$ & $(1,4 \%)^{*}$ & $7 *$ & $(13,7 \%)^{*}$ \\
\hline Total geral & 70 & & 51 & \\
\hline \multicolumn{5}{|c|}{ Teste Qui-quadrado } \\
\hline Graus de liberdade (df) & 3 & & p-valor & 0,062 \\
\hline
\end{tabular}

Fonte: Dados da pesquisa.

Legenda: $*=$ o teste foi realizado com este grupo. 
Tabela 7 - Satisfação

\begin{tabular}{lcc}
\hline Satisfação & $\begin{array}{c}\text { Quantidade de } \\
\text { respondentes }\end{array}$ & \% \\
\hline Satisfeito & 28 & 40,0 \\
Regular & 35 & 50,0 \\
Insatisfeito & 7 & 10,0 \\
\hline
\end{tabular}

Fonte: Dados da pesquisa.

Ao relacionar a informação da satisfação com o objetivo organizacional, não foram encontradas diferenças significativas de satisfação em diferentes objetivos organizacionais. Esses resultados são apresentados na Tabela 8 .
A turbulência ambiental foi medida multiplicando-se os valores atribuídos a cada variável ambiental pelos respondentes, seguindo o método adotado em Gimenez (1993). Esse procedimento resultou em um valor mínimo de 32 , uma mediana de 6.696 e um valor máximo de 62.500 . O valor mediano do índice de turbulência ambiental foi utilizado como critério de classificação do ambiente das empresas estudadas em duas categorias. As empresas, cujo índice de turbulência ambiental situou-se abaixo da mediana, foram classificadas como atuando em um ambiente não turbulento. O outro grupo, composto pelas empresas cujo índice foi maior ou igual à mediana, foi categorizado como atuantes em um ambiente turbulento. Esse procedimento foi adotado para possibilitar as análises sobre a relação entre ambiente, estratégia e desempenho.

Tabela 8 - Satisfação versus objetivo organizacional

\begin{tabular}{|c|c|c|c|c|c|c|c|c|}
\hline \multirow{3}{*}{$\begin{array}{l}\text { Satisfação } \\
\text { Satisfeito }\end{array}$} & \multicolumn{8}{|c|}{ Objetivo organizacional } \\
\hline & \multicolumn{2}{|c|}{ Eficiência interna } & \multicolumn{2}{|c|}{ Eficácia externa } & \multicolumn{2}{|c|}{ Eficácia interna } & \multicolumn{2}{|c|}{ Eficiência externa } \\
\hline & $15^{*}$ & $(38,5 \%)^{*}$ & $8^{*}$ & $(36,4 \%)^{*}$ & 5 & $(62,5 \%)$ & & \\
\hline Regular & $18^{*}$ & $(46,2 \%) *$ & $13^{*}$ & $(59,1 \%) *$ & 3 & $(37,5 \%)$ & 1 & $(100,0 \%)$ \\
\hline Insatisfeito & $6^{*}$ & $(15,4 \%)^{*}$ & $1 *$ & $(4,5 \%)^{*}$ & & & & \\
\hline Totais & 39 & & 22 & & 8 & & 1 & \\
\hline \multicolumn{9}{|c|}{ Teste Qui-quadrado } \\
\hline Graus de liberdade (df) & & 2 & & & & alor & &, 383 \\
\hline
\end{tabular}

Fonte: Dados da pesquisa.

Legenda: $*$ o teste foi realizado com este grupo.

A relação entre turbulência ambiental e estratégia foi testada. O interessante dessa análise é que a estratégia defensiva demonstra-se mais presente em ambientes não turbulentos e a prospectora demonstra-se mais presente em ambientes turbulentos. Esse resultado é consistente com o modelo teórico de Miles e Snow (1978), que argumentam sobre a predominância de empresas prospectoras em ambientes mais dinâmicos, e defensivas em ambientes mais estáveis. Por outro lado, a estratégia analítica mostrou-se uniformemente distribuída nos dois graus de turbulência. A Tabela 9 sintetiza os resultados dessa análise.
Bandeira-de-Mello e Cunha (2004) propuseram que pequenas empresas em ambientes turbulentos e com forte pressão governamental tendem a deixar de lado o lucro e priorizar a sobrevivência. Essa relação entre ambiente e foco estratégico foi testada. Os valores demonstraram que o foco em sobrevivência foi menor nos dois tipos de ambiente. A predominância de respostas indicou um foco estratégico duplo com preocupação de sobrevivência e lucratividade independente da turbulência ambiental, conforme o teste Qui-quadrado ( $\mathrm{p}=0,292 ; \mathrm{df}=1$; $\mathrm{p}$ com correção de Yates $=0,427)$. Os dados são apresentados na Tabela 10. 
Tabela 9 - Turbulência ambiental versus estratégia

\begin{tabular}{|c|c|c|c|c|c|c|c|}
\hline \multirow{3}{*}{$\begin{array}{l}\text { Turbulência } \\
\text { Turbulento }\end{array}$} & \multicolumn{7}{|c|}{ Estratégia } \\
\hline & \multicolumn{2}{|c|}{ Analítica } & \multicolumn{2}{|c|}{ Defensiva } & \multicolumn{2}{|c|}{ Prospectora } & Reativa \\
\hline & $7 *$ & $(53,8 \%)^{*}$ & $8^{*}$ & $(38,1 \%)^{*}$ & $20^{*}$ & $(58,8 \%)^{*}$ & \\
\hline \multirow[t]{2}{*}{ Não turbulento } & $6^{*}$ & $(46,2 \%)^{*}$ & $13^{*}$ & $(61,9 \%)^{*}$ & $14^{*}$ & $(41,2 \%)^{*}$ & $(100,0 \%)$ \\
\hline & 13 & & 21 & & 34 & & 2 \\
\hline \multicolumn{8}{|c|}{ Teste Qui-quadrado } \\
\hline Graus de liberdade (df) & & 2 & & & & alor & 0,322 \\
\hline
\end{tabular}

Fonte: Dados da pesquisa.

Legenda: $*$ o teste foi realizado com este grupo.

Todavia, o fator governamental, que na teoria de Bandeira-de-Mello e Cunha (2004) é visto como um grande gerador de turbulência, não foi tão impactante nas empresas estudadas. Ao contrário, essa foi a quinta variável em termos de pressão ambiental, diferentemente de estudos anteriores, como Miles e Snow (1978), em que essa pressão era a terceira, e no estudo de Gimenez (1993), em que essa pressão era a segunda. Essa diferença de resultados pode se dever à época em que os estudos foram feitos. O estudo de Bandeira-de-Mello

Tabela 10 - Ambiente versus foco estratégico

\begin{tabular}{|c|c|c|c|c|}
\hline \multirow{3}{*}{$\begin{array}{l}\text { Foco estratégico } \\
\text { Lucro }\end{array}$} & \multicolumn{4}{|c|}{ Ambiente } \\
\hline & \multicolumn{2}{|c|}{ Turbulento } & \multicolumn{2}{|c|}{ Não turbulento } \\
\hline & $10^{*}$ & $28,6 \% *$ & $13^{*}$ & $37,1 \% *$ \\
\hline Lucro/sobrevivência & $24 *$ & $68,6 \% *$ & $18^{*}$ & $51,4 \% *$ \\
\hline Sobrevivência & 1 & $2,9 \%$ & 4 & $11,4 \%$ \\
\hline Totais & 35 & & 35 & \\
\hline \multicolumn{5}{|c|}{ Teste Qui-quadrado } \\
\hline \multicolumn{5}{|c|}{ Graus de liberdade (df) $\quad 1$} \\
\hline $\mathrm{p}$-valor & & & & 0,292 \\
\hline p-valor com correção d & e Yate & & & 0,427 \\
\hline
\end{tabular}

Fonte: Dados da pesquisa.

Legenda: $*=$ o teste foi realizado com este grupo. e Cunha foi focado em ambientes com alta regulamentação governamental, ao passo que o estudo de Gimenez foi realizado em uma época de alta incerteza econômica.

Não houve diferença significativa na escolha dos objetivos organizacionais em diferentes pressões ambientais (Teste Qui-quadrado - $\mathrm{p}=0,707 ; \mathrm{df}=2$ ). Também, no que diz respeito à satisfação com o atingimento de objetivos, o teste Qui-quadrado demonstrou que a diferença não é significativa. Os dados estão na Tabela 11.
Tabela 11 - Ambiente versus satisfação

\begin{tabular}{|c|c|c|c|c|}
\hline \multirow[t]{2}{*}{ Satisfação } & \multicolumn{4}{|c|}{ Ambiente } \\
\hline & \multicolumn{2}{|c|}{ Turbulento } & \multicolumn{2}{|c|}{ Não turbulento } \\
\hline Satisfeito & $15^{*}$ & $(42,9 \%)^{*}$ & $13^{*}$ & $(37,1 \%)^{*}$ \\
\hline Regular & $17 *$ & $(48,6 \%)^{*}$ & $18^{*}$ & $(51,4 \%)^{*}$ \\
\hline Insatisfeito & 3 & $(8,6 \%)$ & 4 & $(11,4 \%)$ \\
\hline Totais & 35 & 35 & & \\
\hline \multicolumn{5}{|c|}{ Teste Qui-quadrado } \\
\hline Graus de liberdade (df) & & 1 & & \\
\hline p-valor & & & & 0,693 \\
\hline p-valor com correção d & Yate & & & 0,888 \\
\hline
\end{tabular}

Fonte: Dados da pesquisa.

Legenda: $*=$ o teste foi realizado com este grupo. 
A associação entre objetivo organizacional e estratégia também foi estudada. Os resultados demonstram existir relação entre a escolha da estratégia e o objetivo organizacional desejado, fato comprovado estatisticamente pelo teste Qui-quadrado. Esse resultado corrobora com o estudo de Gimenez (1993), que também comprovou essa relação. Analisando as teorias de Kilmann e Herden (1976) e Miles e Snow (1978), a existência de relação na teoria também é esperada. As empresas que adotaram estratégia defensiva, que tenta manter sua linha de produtos de forma estável sem o foco de tornar-se líder ou inovar, apresentaram em sua maioria o objetivo organizacional de eficácia externa, cujo objetivo é aumentar a satisfação da sociedade com a empresa, diferentemente de outros objetivos organizacionais que envolvem crescimento e inovação, por exemplo. Além disso, o segundo objetivo mais citado para esse tipo de estratégia foi o de eficiência interna, que também é consistente com as idéias de Miles e Snow (1978). Os dados são apresentados na Tabela 12.

No entanto, para a estratégia prospectora, o objetivo mais frequente foi a eficiência interna. Este objetivo é contraditório com o foco da estratégia prospectora, que acentua a busca por inovação, conforme descrito no modelo de Miles e Snow. Esse resultado pode ter sido influenciado pelo fato de que o modelo proposto por Kilmann e Henderman não explicita claramente qual tipo de objetivo organizacional está relacionado com a inovação.
A relação entre estratégia e satisfação não foi comprovada estatisticamente. Da mesma maneira, analisando essa relação em diferentes turbulências ambientais, também não foram encontradas diferenças significativas de satisfação quanto à estratégia.

\section{Conclusão}

Este estudo evidenciou que as pressões ambientais mais impactantes na percepção de dirigentes de micro e pequenas empresas industriais do Paraná foram tecnologia, concorrência e consumidor. Notou-se claramente um enorme crescimento da tecnologia como fator de pressão ambiental, comparando-se com Miles e Snow (1978) e Gimenez (1993). Houve também um decréscimo considerável da percepção de turbulência ambiental sobre as variáveis governo e fornecedores, comparando-se com o estudo de Gimenez (1993).

Esse resultado evidencia que, no contexto competitivo atual das empresas industriais, uma forte pressão ambiental se deve à constante evolução tecnológica presente na maioria dos setores econômicos, seguidos por mudanças de comportamento de concorrentes e consumidores. Essa tendência tem sido comentada por outros estudiosos da gestão estratégica (BANTEL, 1998; ZAHRA; IRELAND; HITT, 2000; PAULA; BIGNETTI, 2003).

Foram encontrados resultados que apoiam o modelo de estratégias genéricas de Miles e Snow (1978).

Tabela 12 - Satisfação versus objetivo organizacional

\begin{tabular}{|c|c|c|c|c|c|c|c|c|}
\hline \multirow{3}{*}{$\begin{array}{l}\text { Objetivo } \\
\text { organizacional }\end{array}$} & \multicolumn{8}{|c|}{ Estratégia } \\
\hline & \multicolumn{2}{|c|}{ Analítica } & \multicolumn{2}{|c|}{ Defensiva } & \multicolumn{2}{|c|}{ Prospectora } & \multicolumn{2}{|c|}{ Reativa } \\
\hline & $10^{*}$ & $(76,9 \%)^{*}$ & 9* & $(42,9 \%)^{*}$ & $18^{*}$ & $(52,9 \%)^{*}$ & 2 & $(100,0 \%)$ \\
\hline Eficácia externa & $1 *$ & $(7,7 \%)^{*}$ & $12 *$ & $(57,1 \%)^{*}$ & $9 *$ & $(26,5 \%)^{*}$ & & \\
\hline Eficácia interna & 2 & $(15,4 \%)$ & & & 6 & $(17,6 \%)$ & & \\
\hline Eficiência externa & & & & & 1 & $(2,9 \%)$ & & \\
\hline Totais & 13 & & 21 & & 34 & & 2 & \\
\hline \multicolumn{9}{|c|}{ Teste Qui-quadrado } \\
\hline Graus de liberdade (df) & & 2 & & & p-valor & 0,024 & & \\
\hline
\end{tabular}

Fonte: Dados da pesquisa.

Legenda: $*$ o teste foi realizado com este grupo. 
Em primeiro lugar, assim como nos estudos anteriores, a pesquisa evidenciou a existência dos quatro tipos de estratégias propostos no modelo de Miles e Snow. Em segundo lugar, a estratégia defensiva mostrou-se mais presente em ambientes menos turbulentos e a prospectora em ambientes mais turbulentos. Esse resultado também é consistente com outros estudos (GIMENEZ et al., 1999; ZAHRA; PEARCE, 1990).

Ademais, o estudo evidenciou uma relação estatisticamente significativa entre estratégia e objetivo organizacional. Esse fato indica que a escolha da estratégia está diretamente ligada ao objetivo desejado pelo estrategista da organização, reforçando o resultado do estudo de Gimenez (1993), que também evidenciou tal relação.

Por outro lado, chama a atenção no estudo a falta de associação significativa entre várias das dimensões analisadas. Esse fato pode sugerir a necessidade de análises multivariadas da variação dos diferentes aspectos envolvidos na administração estratégica de pequenas empresas. Todavia, o tamanho limitado da amostra do estudo não permitiu esse tipo de análise. Dess, Lumpkin e Covin (1997) recomendaram a adoção de modelos configuracionais no estudo da formação de estratégia. Assim, sugere-se que pesquisas futuras procurem avaliar como se dá a relação entre turbulência ambiental, estratégia e desempenho em uma amostra maior que permita análises estatísticas mais robustas.

Por fim, sugere-se que a classificação de foco estratégico desenvolvida neste estudo pode auxiliar acadêmicos na compreensão da administração estratégica nas micro e pequenas empresas. Os resultados e as ferramentas utilizadas na pesquisa podem servir, também, como base ou mesmo como incentivo para novos estudos sobre estratégia em micro e pequenas empresas. Apesar da maioria dos resultados desteestudo não poderem ser considerados conclusivos, o trabalho contribuiu com a evolução da pesquisa sobre estratégia em micro e pequenas empresas no Brasil, ao demonstrar uma relação entre objetivo pessoal do estrategista e estratégia. Este resultado sugere a importância de se investigar a relação entre características e atributos pessoais do estrategista e as escolhas estratégicas feitas no âmbito da micro e pequena empresa.

\section{Referências}

ANSOFF, H. I. A nova estratégia empresarial. Tradução de Antonio Zoratto Sanvicente. 2. ed. São Paulo: Atlas, 1990.
BANDEIRA-DE-MELLO, R.; CUNHA, C. J. C. de A. Administrando o risco: uma teoria substantiva da adaptação estratégica de pequenas empresas a ambientes turbulentos e com forte influência governamental. Revista de Administração Contemporânea, v. 8, n. especial, p. 157-180, 2004.

BANTEL, K. A. Technology-based, "adolescent” firm configurations: strategy identification, context, and performance. Journal of Business Venturing, v. 13, n. 3, p. 205-230, 1998.

BARROS, M. A. Estratégia nas empresas de internet: lições da informalidade. Revista de Administração de Empresas, v. 41, n. 4, p. 32-43, 2001.

CAVEDON, N. R.; FERRAZ, D. L. da S. Representações sociais e estratégia em pequenos comércios. RAE Eletrônica, v. 4, n. 1, art. 14, 2005.

COCHIA, C. B. R.; MACHADO-DA-SILVA, C. L. Ambiente, interpretação e estratégia em organizações paranaenses dos setores de vestuário e alimentos. Revista de Administração Contemporânea, v. 8, n. especial, p. 11-35, 2004.

COOPER, A. C. Strategic management: new ventures and small firms. Long Range Planning, v. 14, n. 5, p. 39-45, 1981.

CORRÊA, H. L.; PROCHNO, P. J. L. de C. Desenvolvimento de uma estratégia de manufatura em ambiente turbulento. Revista de Administração de Empresas, v. 38, n. 1, p. 64-79, 1998.

DAVIG, W. Business strategies in smaller manufacturing firms. Journal of Small Business Management, v. 24, n. 1, p. 38-46, 1986.

DESS, G. G.; LUMPKIN, G. T.; COVIN, J. G. Entrepreneurial strategy making and firm performance: tests of contingency and configurational models. Strategic Management Journal, v. 18, n. 9, p. 677-695, 1997.

DOWNEY, H. K.; HELLRIEGEL, D.; SLOCUM, J. W. The effects of individual differences on managers' perceptions of environment uncertainty. Academy of Management Proceedings, v. 25, p. 200-202, 1975.

GIMENEZ, F. A. P. Estratégia e criatividade em pequenas empresas. Revista de Administração, v. 28, n. 2, p. 72-82, 1993.

GIMENEZ, F. A. P. Escolhas estratégicas e estilo cognitivo: um estudo com pequenas empresas. Revista de Administração Contemporânea, v.2,n.1,p.27-45, 1998. 
GIMENEZ, F. A. P. et al. Estratégia em pequenas empresas: uma aplicação do modelo de Miles e Snow. Revista de Administração Contemporânea, v. 3, n. 2, p. 53-74, 1999.

GIMENEZ, F. A. P. O estrategista na pequena empresa. Maringá: Edição do autor, 2000.

HAYASHI Jr., P.; BARABIUK, J. A.; BULGACOV, S. Mudanças de conteúdo estratégico em pequenas empresas de massas alimentícias. Revista de Administração Contemporânea, v. 10, n. 3, p. 159-179, 2006.

HOY, F.; HELLRIEGEL, D. The Kilmann and Herden model of organizational effectiveness criteria for small business managers. Academy of Management Journal, v. 25, n. 2, p. 308-322, 1982.

JAUCH, L. R.; KRAFT, K. L. Strategic management of uncertainty. Academy of Management Review, v. 11, n. 4, p. 777-790, 1986.

KILMANN, R. H.; HERDEN, R. P. Towards a systemic methodology for evaluating theimpact of interventions on organizational effectiveness. Academy of Management Review, v. 28, p. 87-98, 1976.

MACHADO, H. V. (Org.). Causas de mortalidade de pequenas empresas: coletânea de estudos. Maringá: EDUEM, 2007.

MILES, R. E.; SNOW, C. C. Organizational strategy, structure, and process. 2nd ed. New York: McGraw-Hill, 1978.

MILLIKEN, F. J. Three types of perceived uncertainty about the environment: state, effect, and response uncertainty. Academy of Management Review, v. 12, n. 1, 133-143, 1987.

MINTZBERG, H.; QUINN, J. B. O processo da estratégia. Tradução de James Sunderland Cook. 3. ed. Porto Alegre: Bookman, 2001.

PAULA, F. de O. Fatores que influenciam o desempenho das firmas brasileiras: um modelo integrativo. 2006. 255 f. Dissertação (Programa de Pós-Graduação em Administração) - Pontifícia Universidade Católica do Rio de Janeiro, Rio de Janeiro, 2006.

PAULA, E. W.; BIGNETTI, L. P. Estudo sobre as práticas de inovação e as estratégias adotadas por empreendedores de alta tecnologia. Economia e Gestão, v. 3, n. 6, p. 42-57, 2003.

PLESHKO, L. P. Strategic organization, organizational structure, and the associated effects on performance. Journal of Financial Services Marketing, v. 12, n. 1, p. 53-64, 2007.
PORTER, M. E. A nova era da estratégia. In: JÚLIO, C. A.; SALIBI NETO, J. (Org.). Estratégia e planejamento: autores e conceitos imprescindíveis. 2. ed. São Paulo: PubliFolha, 2002. p. 21-38.

RAMOS, S. C.; GIMENEZ, F. A. P.; FERREIRA, J. M. O papel da análise de concorrência na formulação da estratégia em pequenas empresas. In: ENCONTRO DE ESTUDOS SOBRE EMPREENDEDORISMO E GESTÃO DE PEQUENAS EMPRESAS, 4., 2005, Curitiba. Anais... Curitiba; Maringá; Londrina: PUCPR; UEM; UEL, 2005.

ROBINSONJr., R. B.; PEARCEII,J. A. Research thrusts in small firm strategic planning. Academy of Management Review, v. 9, n. 1, p. 128-139, 1984.

ROSSETTO, C. R. et al. Comportamento estratégico em pequenas e médias empresas - estudo multi-casos em pousadas na Lagoa da Conceição - Florianópolis - SC. Revista de Negócios, v. 11, n. 3, p. 93-108, 2006.

SANTOS, L. L. da S.; ALVES, R. C.; ALMEIDA, K. N. T. de. Formação de estratégia nas micro e pequenas empresas: um estudo no Centro-Oeste mineiro. Revista de Administração de Empresas, v. 47, n. 4, p. 59-73, 2007.

SERVIÇO BRASILEIRO DE APOIO ÀS MICRO E PEQUENAS EMPRESAS - SEBRAE. MPE em números. Disponível em: <www.sebrae.com.br>. Acesso em: 1 ago. 2007.

THOMPSON, J. D. Organization in action. 2nd ed. New York: McGraw-Hill, 1967.

ZAHRA, S. A.; IRELAND, R. D.; HITT, M. A. International expansion by new venture firms: international diversity, mode of market entry, technological learning and performance. Academy of Management Journal, v. 43, n. 5, p. 925-950, 2000.

ZAHRA, S. A.; PEARCE II, J. A. Research evidence on the Miles-Snow typology. Journal of Management, v. 16, n. 4, p. 751-768, 1990.

Recebido: 23/09/2010

Received: 09/23/2010

Aprovado: $15 / 10 / 2010$ Approved: 10/15/2010 